\title{
Wakefields in conducting waveguides with a lossy dielectric channel
}

\author{
M. I. Ivanyan $\odot,{ }^{1,}{ }^{*}$ L. V. Aslyan $\odot,{ }^{1}$ K. Floettmann, ${ }^{2}$ F. Lemery, ${ }^{2}$ and V. M. Tsakanov ${ }^{1,3}$ \\ ${ }^{1}$ CANDLE Synchrotron Research Institute, 31 Acharyan, 0040 Yerevan, Armenia \\ ${ }^{2}$ DESY, Notkestrasse 85, 22603 Hamburg, Germany \\ ${ }^{3}$ Yerevan State University, 1 Alex Manoogyan, 0025 Yerevan, Armenia
}

(Received 21 January 2020; accepted 1 April 2020; published 22 April 2020)

\begin{abstract}
The resonant properties of the impedance of a two-layer circular metal-dielectric waveguide are investigated by taking into account the finite conductivity of the metallic pipe and the dissipation of the radiated energy in the dielectric layer. The dispersion relations for azimuthally symmetric monopole TM modes are analyzed. It is shown that, for an outer metallic layer with finite conductivity and a thin internal dielectric cover, the waveguide has only a single slowly propagating $\mathrm{TM}_{01}$ mode. The point charge longitudinal wake potentials are evaluated for thin and thick internal layers.
\end{abstract}

DOI: $10.1103 /$ PhysRevAccelBeams.23.041301

\section{INTRODUCTION}

Metal-dielectric [1-25], bimetallic [26-28], and corrugated [29-32] structures have attracted strong interest for particle radiation, acceleration, and beam manipulation in the past decades. Dielectric loaded structures are promising for beam-based terahertz generation [6-12], two-beam acceleration [13-16], beam diagnostics [17], and beam manipulation [18-21]. Recently, it was demonstrated that the incorporation of a dielectric-lined waveguide located downstream of a photoinjector gun can provide a sufficient energy modulation from the self-wake to ballistically bunch a beam on a meter-scale footprint [22]. Note that a thin internal dielectric layer also serves as a model for the mathematical description of corrugated and rough inner surfaces of accelerating structures [29-32]. The special case of an aluminum pipe with a dielectric aluminum oxide layer has been studied in Ref. [25], where the effects of an oxide layer on the resistive-wall wakefields have been evaluated numerically by means of a finite element method.

Studies of metal-dielectric structures suffer often from a gap between theory and practical conditions in the experiment, because simplified models are employed which allow only a qualitative interpretation of the experimental results. Usually, in the study of the dielectric loaded waveguides, the external metal wall is assumed to be an ideal conductor, and electromagnetic energy losses in the dielectric layer are neglected. In this case, the structure impedance contains only an imaginary part, which contains an infinite number

\footnotetext{
*ivanian@asls.candle.am

Published by the American Physical Society under the terms of the Creative Commons Attribution 4.0 International license. Further distribution of this work must maintain attribution to the author(s) and the published article's title, journal citation, and DOI.
}

of alternating divergent peaks, which are equally spaced at the resonance frequencies (cf. Fig. 13, left), while the wake function turns into an infinite sum of undamped cosinelike functions. Note that the impedance in this model has an infinite number of excited synchronous modes for any thickness of the dielectric layer. Single-mode radiation can then be achieved only by using an extended bunch length, providing exponential damping of high-order modes $[4,5]$.

In this paper, the longitudinal impedance and the point wake potential for a finite conductivity metallic waveguide with a lossy internal dielectric layer are studied. It is shown that, under certain conditions, the finite conductivity of the outer layer leads to a new dispersion relation for Cherenkov radiation that is characterized by a single synchronous $\mathrm{TM}_{01}$ mode. The resistivity of the outer wall substantially changes the configuration of the impedance curve: it does not contain divergences, acquires a real component, and, with a sufficiently thin dielectric layer, becomes single resonant with the canonical form of a narrow-band impedance. The wake function of the point charge is then monochromatic. The main differences between the processes of radiation in a metal-dielectric waveguide with a thin and a thick internal dielectric layer are examined. We also evaluate the modifications of the impedance and wakefields due to a finite metallic conductivity and a lossy dielectric.

\section{ANALYTICAL MODEL OF IMPEDANCE AND DISPERSION RELATION}

Consider a point charge $q$ moving along the axis of a cylindrical metallic waveguide with an internal dielectric layer (Fig. 1), where $a_{1}$ is the inner radius of the waveguide, $a_{2}>a_{1}$ is the inner radius of the metallic wall, $d=a_{2}-a_{1}$ is the dielectric layer thickness, and $a_{3}$ is the outer radius of the waveguide. 


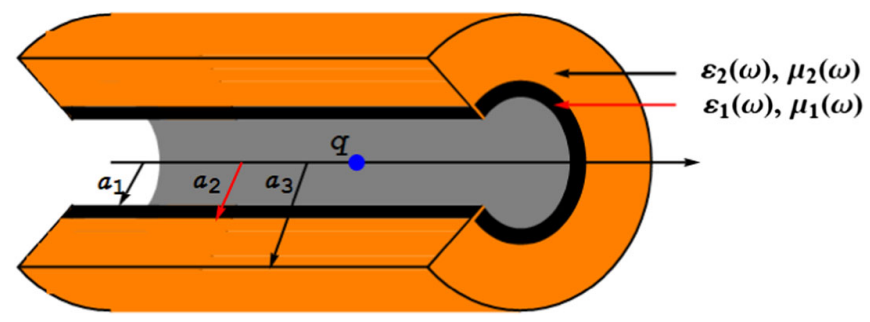

FIG. 1. Round two-layer metal-dielectric waveguide.

For numerical simulations, we will assume a copper waveguide with a conductivity of $\sigma_{2}=58 \times 10^{6} \Omega^{-1} \mathrm{~m}^{-1}$. The dielectric constant of the conducting outer layer is given by $\varepsilon_{2}=1+j \sigma_{2} / \varepsilon_{0} \omega$, where $\varepsilon_{0}$ is the vacuum dielectric constant and $\omega$ is the angular frequency. The real part $\varepsilon_{1}^{\prime}$ of the relative dielectric constant $\varepsilon_{1}=\varepsilon_{1}^{\prime}+j \varepsilon_{1}^{\prime \prime}$ of the dielectric layer is assumed to be $\varepsilon_{1}^{\prime}=10$, while the imaginary part will vary as $\varepsilon_{1}^{\prime \prime}=0,0.1,0.5$, and 3.0. As an example of materials for a dielectric layer with $\varepsilon_{1}^{\prime}=10$, some grades of condensed ceramics, micas furan resin, melamine resin, urea resin, and glasses can be considered $[33,34]$. In the framework of our study, we assume the materials of both layers are nonmagnetic: $\mu_{1}=\mu_{2}=1$.

The inner radius of the waveguide is $a_{1}=2 \mathrm{~mm}$. Two values of the thickness of the dielectric layer are

considered: relatively thick $d=200 \mu \mathrm{m}$ and rather thin $d=2 \mu \mathrm{m}$. We assume an infinite outer wall thickness, which is valid for the outer layer thickness much larger than the skin depth, an assumption generally satisfied for all practical cases when considering a copper wall.

Exact formulas for the impedances of infinite homogeneous cylindrical waveguides with laminated walls can be obtained using the matrix formalism [35]. The current study is based on the exact expression for the longitudinal monopole impedance of a cylindrical waveguide with a two-layer wall. For an ultrarelativistic particle moving along the axis of the waveguide with a speed $v$ equal to the speed of light $c$ in a vacuum, the longitudinal impedance is expressed as [35]

$$
Z_{\|}=j \frac{Z_{0}}{\pi k a_{1}^{2}}\left\{1-\frac{2 \varepsilon_{1}}{a_{1} \beta_{1}} G\right\}^{-1}
$$

where

$$
G=\frac{\beta_{2} \varepsilon_{1} U_{4}+\beta_{1} \varepsilon_{2} U_{3} \alpha}{\beta_{2} \varepsilon_{1} U_{2}+\beta_{1} \varepsilon_{2} U_{1} \alpha}, \quad \alpha=\frac{K_{1}\left(a_{2} \beta_{2}\right)}{K_{0}\left(a_{2} \beta_{2}\right)},
$$

$$
\begin{aligned}
& U_{1}=I_{0}\left(\beta_{1} a_{2}\right) K_{0}\left(\beta_{1} a_{1}\right)-I_{0}\left(\beta_{1} a_{1}\right) K_{0}\left(\beta_{1} a_{2}\right) \approx \sinh \left(\beta_{1} d\right) / \beta_{1} \sqrt{a_{1} a_{2}}, \\
& U_{2}=I_{1}\left(\beta_{1} a_{2}\right) K_{0}\left(\beta_{1} a_{1}\right)+I_{0}\left(\beta_{1} a_{1}\right) K_{1}\left(\beta_{1} a_{2}\right) \approx \cosh \left(\beta_{1} d\right) / \beta_{1} \sqrt{a_{1} a_{2}}, \\
& U_{3}=-I_{0}\left(\beta_{1} a_{2}\right) K_{1}\left(\beta_{1} a_{1}\right)-I_{1}\left(\beta_{1} a_{1}\right) K_{0}\left(\beta_{1} a_{2}\right) \approx-\cosh \left(\beta_{1} d\right) / \beta_{1} \sqrt{a_{1} a_{2}}, \\
& U_{4}=I_{1}\left(\beta_{1} a_{1}\right) K_{1}\left(\beta_{1} a_{2}\right)-I_{1}\left(\beta_{1} a_{2}\right) K_{1}\left(\beta_{1} a_{1}\right) \approx-\sinh \left(\beta_{1} d\right) / \beta_{1} \sqrt{a_{1} a_{2}}
\end{aligned}
$$

with the impedance of free space $Z_{0}=120 \pi \Omega$, the modified Bessel functions of the first and second kind and zero and first order $I_{0,1}, K_{0,1}$, the transverse wave numbers for the materials of the first and second layers $\beta_{1,2}=k \sqrt{1-\varepsilon_{1,2} \mu_{1,2}}$, and the wave number $k=\omega / c$. Note that, for a high conductivity of the outer wall, $\alpha \approx 1$. The approximations in Eq. (3) are valid for $\left|\beta_{1}\right| a_{1} \gg 1$. By substituting the approximate expressions into Eq. (2), we can obtain the short-wave asymptotic representation for the impedance (1) (compare with Ref. [32]):

$Z_{\|}=j \frac{Z_{0}}{\pi k a_{1}^{2}}\left\{1+\frac{2 \varepsilon_{1}}{a_{1} \beta_{1}} \frac{\operatorname{th}\left(\beta_{1} d\right)+u}{1+u \operatorname{th}\left(\beta_{1} d\right)}\right\}^{-1}, \quad u=\frac{\beta_{1} \varepsilon_{2}}{\beta_{2} \varepsilon_{1}}$.

The factor $G$ [Eq. (2)] can be divided into two terms, the first of which contains only the parameters of the internal dielectric layer and the second of which vanishes as the conductivity of the outer metal layer tends to infinity:

$$
G=\left(\frac{U_{3}}{U_{1}}+\frac{1}{\beta_{1}^{2} a_{1} a_{2}} \frac{\varepsilon_{1}}{U_{1}} \frac{1}{\varepsilon_{1} U_{2}+\beta_{1} U_{1} \frac{\varepsilon_{2}}{\beta_{2}} \alpha}\right)
$$

In deriving Eq. (5), the following relation has been used:

$$
U_{4} U_{1}-U_{3} U_{2}=\frac{1}{\beta_{1}^{2} a_{1} a_{2}} .
$$

The impedance [Eq. (1)] can now be written as a sum of two parallel connected resistances: $Z_{\text {diel }}$ and $Z_{\text {res }}$. The first describes the impedance of a metal-dielectric waveguide with an ideally conducting outer wall, and the second one takes into account the contribution of the finite conductivity of the outer wall:

$$
\begin{gathered}
Z_{\|}=\left\{Z_{\text {diel }}^{-1}+Z_{\text {res }}^{-1}\right\}^{-1}, \\
Z_{\text {diel }}=j \frac{Z_{0}}{\pi k a_{1}^{2}}\left[1-\frac{2 \varepsilon_{1}}{a_{1} \beta_{1}} \frac{U_{3}}{U_{1}}\right]^{-1},
\end{gathered}
$$




$$
Z_{\mathrm{res}}=-j \frac{Z_{0}}{2 \pi k \varepsilon_{1}} \beta_{1}^{3} a_{2} U_{1}\left(U_{2}+\frac{\beta_{1}}{\varepsilon_{1}} \frac{\varepsilon_{2}}{\beta_{2}} U_{1} \alpha\right) .
$$

Note that the resistive part of the impedance [Eq. (9)] is included as a parallel additive to its purely dielectric component [Eq. (8)]. Its contribution, besides the outer layer conductivity $\sigma_{2}$, depends on the parameters of the internal dielectric layer $\varepsilon_{1}, a_{1}$, and $a_{2}$. This term is due to the energy dissipation in the metal wall modified by the dielectric layer.

If the outer wall of the waveguide is an ideal conductor $\left(\sigma_{2} \rightarrow \infty\right)$, expression (7) turns to $Z_{\text {diel }}(8)$ :

$$
Z_{\|}=Z_{\text {diel }}
$$

In the alternative limiting case $\varepsilon_{1} \rightarrow \varepsilon_{0}$, expression (7) tends to the impedance of an unbound single-layer resistive tube [36] with inner radius $a_{2}$ :

$Z_{\|}=\tilde{Z}_{\text {res }}=j \frac{Z_{0}}{\pi k a_{2}}\left(\frac{2 \varepsilon_{2}}{\beta_{2}}+a_{2}\right)^{-1} \approx \frac{Z_{0} s_{0}}{2 \pi a_{2}^{2}}\left(\frac{1-j}{\sqrt{\kappa}}+\frac{j \kappa}{2}\right)^{-1}$.

Here $\kappa=k s_{0}$ is the dimensionless wave number, and $s_{0}=\left(\frac{2 a_{2}^{2}}{Z_{0} \sigma_{2}}\right)^{1 / 3}$ is a characteristic distance. In the case of $\varepsilon_{1}^{\prime \prime}=0$, Eq. (8) corresponds to the perfectly conducting waveguide with a lossless internal dielectric layer.

The dispersion relation connects the longitudinal wave number $p$ with the frequency $p=\sqrt{k^{2}-\nu_{0}^{2} / a_{1}^{2}}$, where $\nu_{0}$ is the desired mode-dependent dimensionless transverse wave number of the TM mode in the vacuum region. The transverse wave numbers of all modes are obtained using the field-matching conditions at the boundaries of the layers, which for the azimuthally symmetric modes $\mathrm{TM}_{0 i}$ $(i=1,2,3 \ldots)$ is given by the relation [37]

$\frac{\varepsilon_{1} \nu_{0} J_{0}\left(\nu_{0}\right) W_{3}+\nu_{1} J_{1}\left(\nu_{0}\right) W_{1}}{\varepsilon_{1} \nu_{0} J_{0}\left(\nu_{0}\right) W_{4}+\nu_{1} J_{1}\left(\nu_{0}\right) W_{2}}+\frac{\varepsilon_{1} \nu_{2}}{\varepsilon_{2} \nu_{1}} \frac{H_{0}^{(1)}\left(\xi \nu_{2}\right)}{H_{1}^{(1)}\left(\xi \nu_{2}\right)}=0$

with the initial conditions $\nu_{0} \rightarrow \chi_{0 i}(i=1,2,3 \ldots)$ for $k \rightarrow 0$, where $\chi_{0 i}$ is the $i$ th root of the zero-order Bessel function $\left[J_{0}\left(\chi_{0 i}\right)=0\right]$. Together with the continuity requirement of the function $\nu_{0}(k)$, the above initial conditions uniquely determine the transverse wave numbers $\nu_{0 i}$ of the $\mathrm{TM}_{0 i}$ modes and the corresponding dispersion relations.

In the lossy structures under consideration, the transverse $\nu_{0 i}$ and longitudinal $p$ wave numbers are complex quantities. In this case, the transverse propagation constant is essentially the real component of the transverse wave number $\operatorname{Re}\{p\}$, while its imaginary component $\operatorname{Im}\{p\}$ is responsible for the attenuation of the wave. Thus, the condition for synchronization of the phase velocity of the waveguide mode with the velocity of an ultrarelativistic particle is the equality $k=\operatorname{Re}\{p\}$ (Figs. 4 and 9) or $\Delta k=$ $k-\operatorname{Re}\{p\}=0$ (Fig. 11).

In Eq. (12), $\xi$ is the ratio $a_{2} / a_{1}$, and $\nu_{1}$ and $\nu_{2}$ are the transverse wave numbers of the same mode in the inner layer and in the outer wall, respectively:

$$
\nu_{1,2}=\sqrt{k^{2} a_{1}^{2}\left(\varepsilon_{1,2} \mu_{1,2}-1\right)+\nu_{0}^{2}} .
$$

$J_{0,1}$ and $H_{0,1}^{(1)}$ are Bessel and Hankel functions of the first kind and zero and first order, respectively:

$$
\begin{aligned}
& W_{1}=H_{0}^{(1)}\left(\nu_{1}\right) J_{0}\left(\xi \nu_{1}\right)-J_{0}\left(\nu_{1}\right) H_{0}^{(1)}\left(\xi \nu_{1}\right), \\
& W_{2}=-H_{0}^{(1)}\left(\nu_{1}\right) J_{1}\left(\xi \nu_{1}\right)+J_{0}\left(\nu_{1}\right) H_{1}^{(1)}\left(\xi \nu_{1}\right), \\
& W_{3}=-H_{1}^{(1)}\left(\nu_{1}\right) J_{0}\left(\xi \nu_{1}\right)+J_{1}\left(\nu_{1}\right) H_{0}^{(1)}\left(\xi \nu_{1}\right), \\
& W_{4}=H_{1}^{(1)}\left(\nu_{1}\right) J_{1}\left(\xi \nu_{1}\right)-J_{1}\left(\nu_{1}\right) H_{1}^{(1)}\left(\xi \nu_{1}\right) .
\end{aligned}
$$

With an ideally conducting outer wall $\left(\varepsilon_{2} \rightarrow \infty\right)$, Eq. (12) is simplified to

$$
\varepsilon_{1} \nu_{0} J_{0}\left(\nu_{0}\right) W_{3}+\nu_{1} J_{1}\left(\nu_{0}\right) W_{1}=0
$$

\section{LONGITUDINAL IMPEDANCE AND WAKE FUNCTION-THICK INNER LAYER}

Figure 2 presents graphs of the longitudinal impedance of a metal-dielectric tube with a thick $(d=200 \mu \mathrm{m})$ internal dielectric coating with relatively small values of the imaginary part of the dielectric constant $\left(\varepsilon_{1}^{\prime \prime}=0,0.1\right.$, and 0.5). Figure 3 presents the impedance for the case of a relatively large imaginary part of the dielectric constant $\left(\varepsilon_{1}^{\prime \prime}=3\right)$.

The impedances (Fig. 2) are characterized by multiple resonant frequencies. In the cases presented in Figs. 2 and 3 , the ratio $d / a_{1}=0.1$ is small. In accordance with this, the resonance values of the impedances rather rapidly decrease with increasing frequencies of the resonances, even at $\varepsilon_{1}^{\prime \prime}=0$. In the latter case, the attenuation is solely due to the resistivity of the outer wall material. As one can see, even such a highly conductive material as copper leads to a significant attenuation.

In Fig. 4, the dispersion curves for the first three modes $\left(\mathrm{TM}_{0 i}, i=1,2,3\right)$ are presented for different values of the imaginary component of the dielectric constant of the inner coating. Note that the synchronous frequencies (the phase velocity is equal to the velocity of light) correspond to the resonant frequencies of the longitudinal impedance in Fig. 2. The effect of higher resonant frequencies on the 

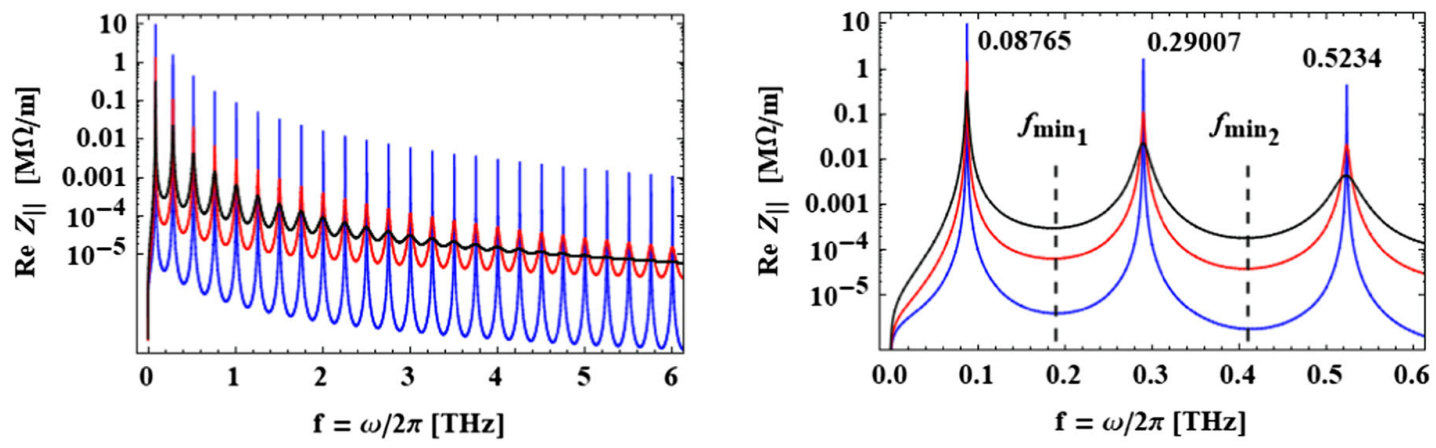

FIG. 2. The real part of longitudinal monopole impedance of a two-layer metal-dielectric waveguide for $\varepsilon_{1}^{\prime}=10$ and for three different values of $\varepsilon_{1}^{\prime \prime}: \varepsilon_{1}^{\prime \prime}=0$ (blue line), 0.1 (red line), and 0.5 (black line). Left: Wide terahertz range of impedance distribution. Right: The resonant frequencies of the first three extremes of the same resonance curve are shown; $f_{\min _{1}}$ and $f_{\min _{2}}$ are the local minima of the real part of impedance, separating the contributions of individual modes.

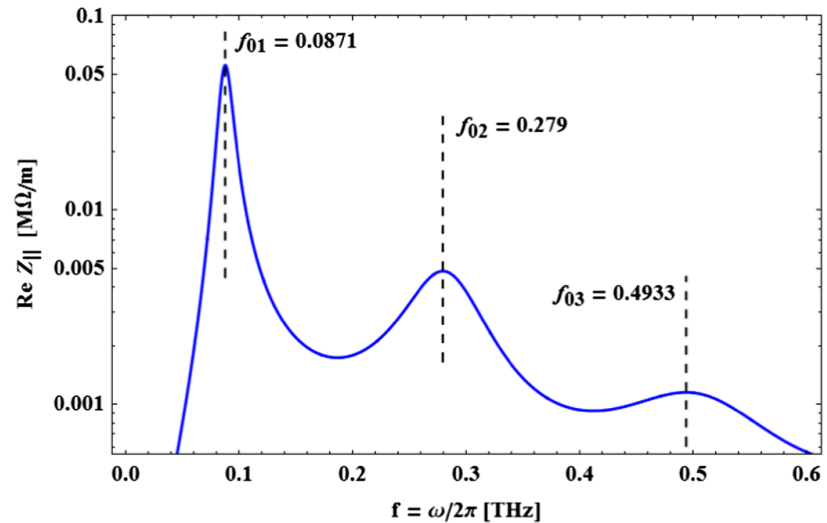

FIG. 3. The real part of longitudinal monopole impedance of a two-layer metal-dielectric waveguide for $a_{1}=2 \mathrm{~mm}, d=200 \mu \mathrm{m}$, $\varepsilon_{1}^{\prime}=10$, and $\varepsilon_{1}^{\prime \prime}=3$.

test particle can be evaluated by studying the shape of the corresponding wake functions, presented in Fig. 5.

Hereinafter, the wake function is calculated as the inverse Fourier transform of the impedance (1):

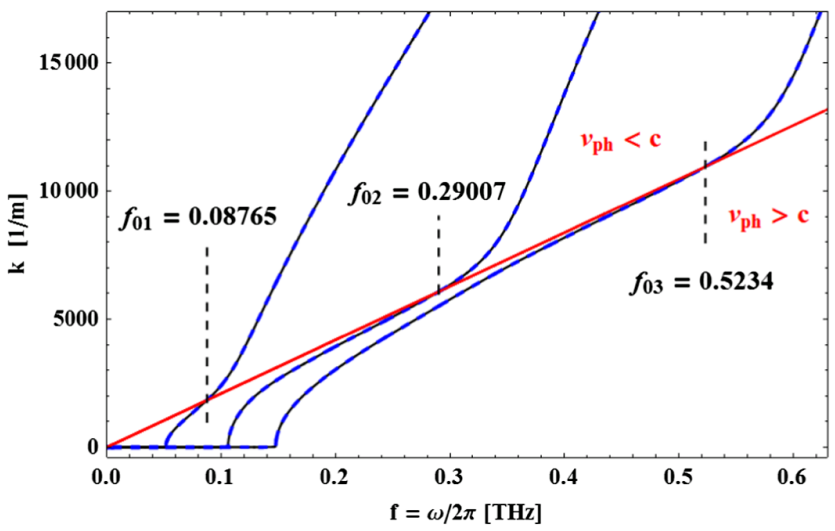

FIG. 4. Overlapping dispersion curves of the first three TM modes of a metal-dielectric waveguide for a lossless $\varepsilon_{1}^{\prime \prime}=0$ (blue, dashed line) and low-loss $\varepsilon_{1}^{\prime \prime}=0.5$ (black, solid line) dielectric.

$$
W_{\|}=\frac{1}{2 \pi} \int_{-\infty}^{\infty} Z_{\|} e^{-j s \omega / c} d \omega,
$$

where $s$ is the distance to the test particle. The calculations are performed numerically, and the area of integration is gradually increased, which leads to the vanishing of the wake function in front of the leading ultrarelativistic charge $(s<0)$ due to the causality principle.

The distortion imposed by the higher resonant frequencies is clearly visible in Fig. 5; however, they weaken as the imaginary part of the dielectric constant increases due to damping. In particular, with $\varepsilon_{1}^{\prime \prime}=0.5$, the effect of the higher-order resonances appears only in the immediate vicinity of the particle (with $s<10 \mathrm{~mm}$ ). Further away, the damped cosinelike curve clearly arises, due to the prevailing contribution of the fundamental $\mathrm{TM}_{01}$ mode.

In Fig. 6, the contributions of the first three resonant frequencies to the wake function for the cases $\varepsilon_{1}^{\prime \prime}=0$ and $\varepsilon_{1}^{\prime \prime}=0.5$ are separately highlighted.

In the first case (no attenuation, $\varepsilon_{1}^{\prime \prime}=0$ ), all three harmonics make a significant contribution to the total wake function. The sum of the contributions of the first three harmonics (Fig. 7) basically repeats the form of the full wake function (Fig. 5, blue curve), except for the immediate vicinity of the particle.

In the second case $\left(\varepsilon_{1}^{\prime \prime}=0.5\right)$, the convergence of the harmonics to the full wake function is faster. Mainly the first two harmonics participate in the formation of the wake function (Fig. 8).

Note that higher-order harmonics need to be taken into account for numerical simulations to assure the causality principle, i.e., the vanishing of the field ahead of an ultrarelativistic charge $(s<0)$.

For relatively large attenuation values as $\varepsilon_{1}^{\prime \prime}=3$ (Fig. 3), the situation becomes fundamentally different. While multiple resonance peaks are still present in the impedance (Fig. 3), only the main resonance frequency turns out to be in phase with the particle velocity (Fig. 9, left). The dispersion curves of the higher harmonics approach the 


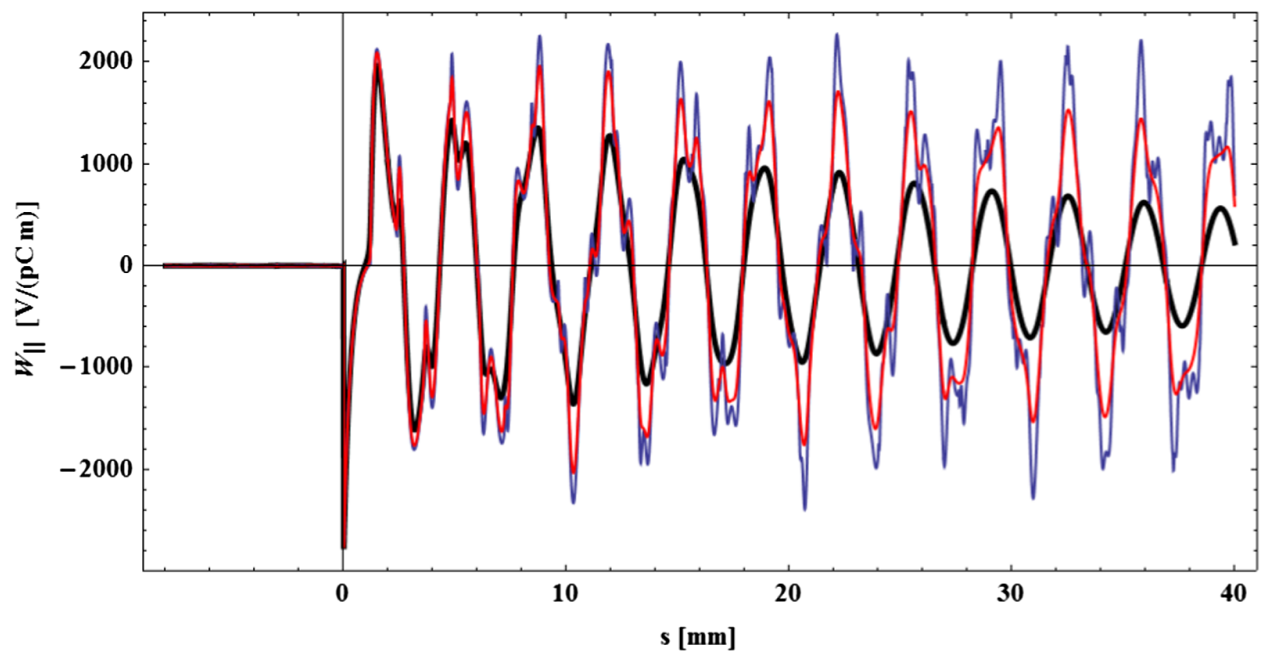

FIG. 5. Longitudinal monopole wake function of the considered bilayer metal-dielectric structure at various values of $\varepsilon_{1}^{\prime \prime}: \varepsilon_{1}^{\prime \prime}=0$ (blue line), $\varepsilon_{1}^{\prime \prime}=0.1$ (red line), and $\varepsilon_{1}^{\prime \prime}=0.5$ (black line).
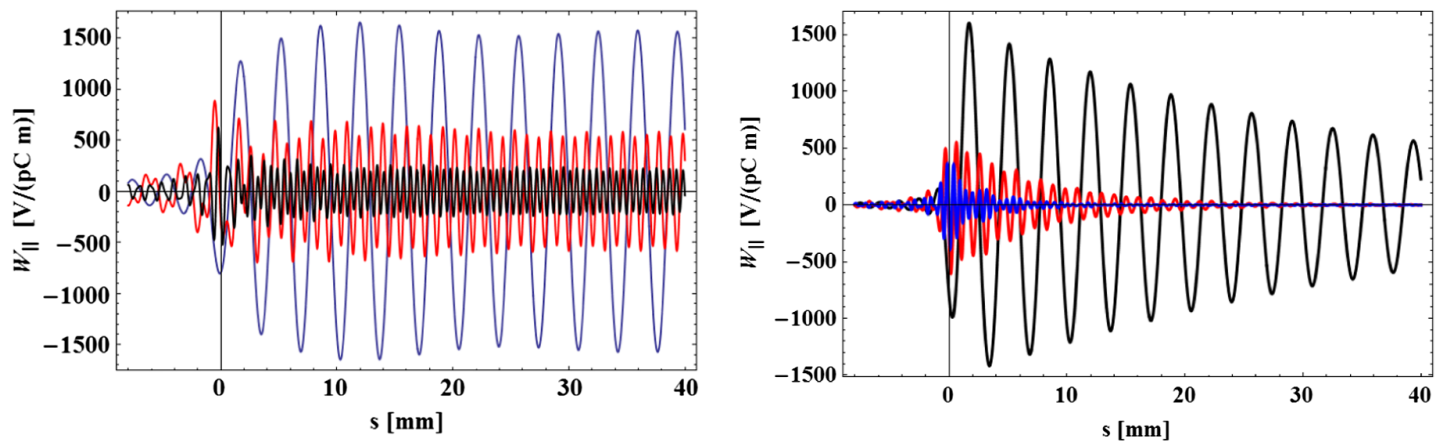

FIG. 6. Separated contributions of the first three resonances to the wake function: $\varepsilon_{1}^{\prime \prime}=0$ (left) and $\varepsilon_{1}^{\prime \prime}=0.5$ (right).

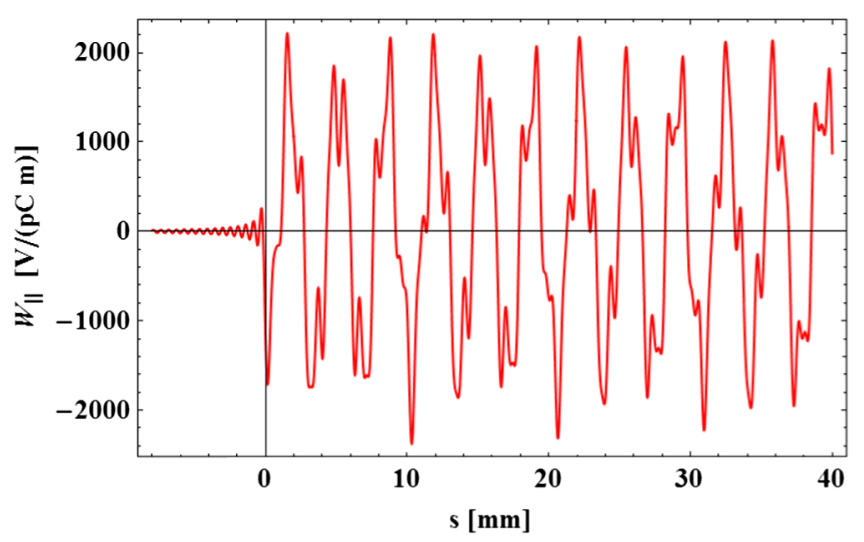

FIG. 7. The total contribution of the first three resonances to the wake function for $\varepsilon_{1}^{\prime \prime}=0$.

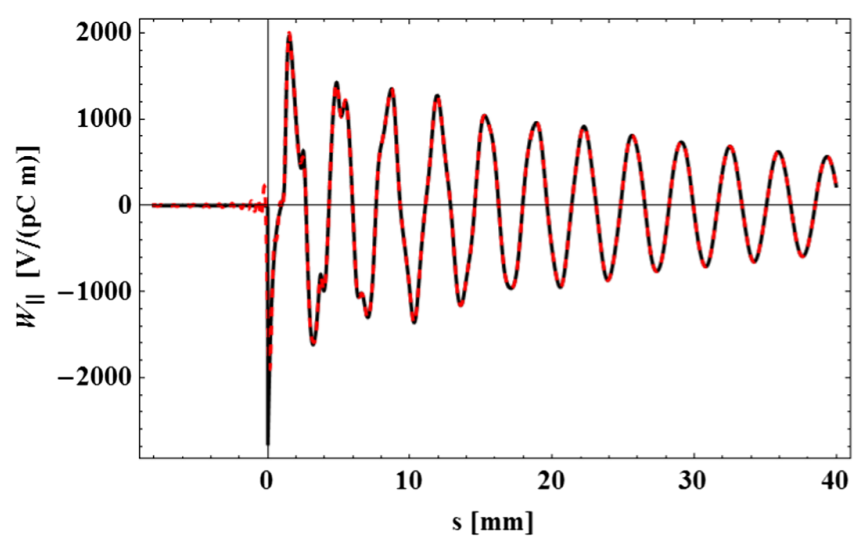

FIG. 8. Comparison of the sum of the first two resonance contributions to the wake function (red, dashed line) with the full wake function (black, solid line) for $\varepsilon_{1}^{\prime \prime}=0.5$. 

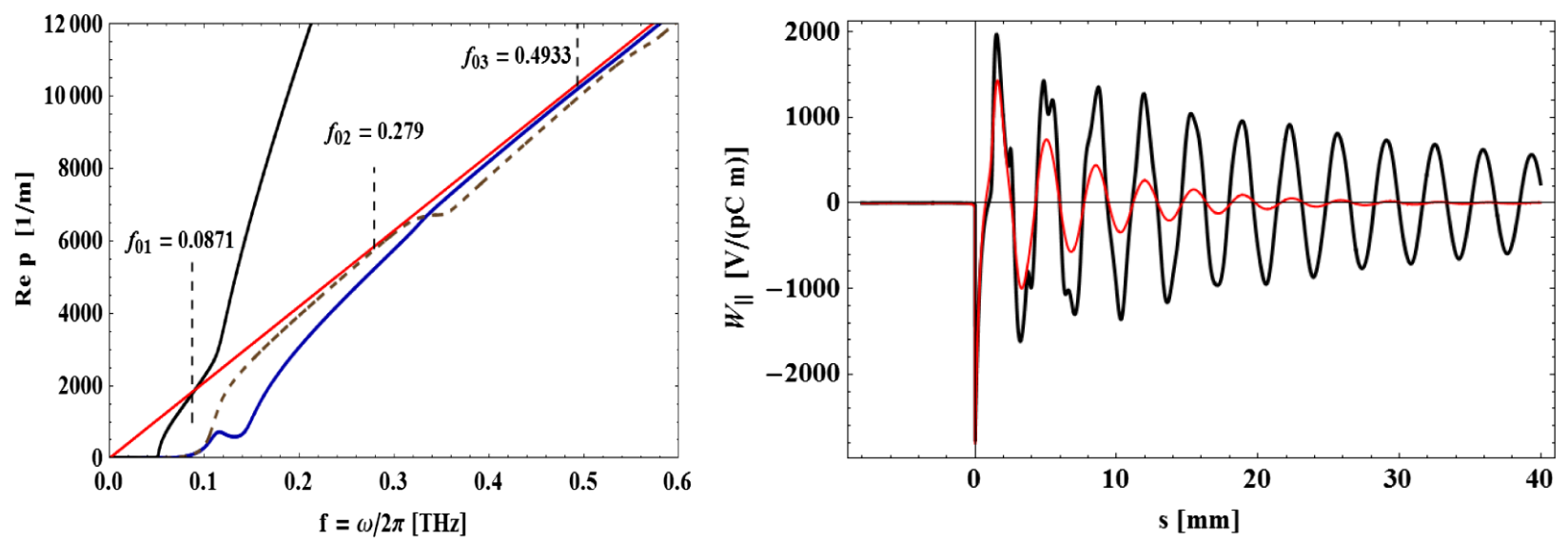

FIG. 9. Two-layer metal-dielectric waveguide for the case $\varepsilon_{1}^{\prime \prime}=3$. Dispersion curves (left) for the $\mathrm{TM}_{01}$ (black, solid line), $\mathrm{TM}_{02}$ (black, dashed line), and $\mathrm{TM}_{03}$ (blue, solid line) modes and longitudinal wake function (right) for $\varepsilon_{1}^{\prime \prime}=3$ (red line) and for $\varepsilon_{1}^{\prime \prime}=0.5$ (black line, for comparison).

speed of light line but do not cross it. Thus, the phase velocities of the higher harmonics turn out to be greater than the speed of light; i.e., they are rapidly propagating. As a result, the wake function becomes harmonious, although it decreases rapidly (Fig. 9, right).

\section{LONGITUDINAL IMPEDANCE AND WAKE FUNCTION-THIN DIELECTRIC LAYER}

As demonstrated in the previous section, the metaldielectric structure with a relatively thick dielectric inner layer $\left(d / a_{1}=0.1\right)$ is a multimode structure from the point of view of particle radiation. Note that, for the ideally conducting outer layer, the structure is also multimodal for any thickness of the inner dielectric layer. However, for a finite conductivity of the outer layer, the thin inner dielectric layer can lead to a single-mode structure, where only the fundamental $\mathrm{TM}_{01}$ mode can be excited. The following section provides an example of such a structure with a significantly smaller thickness of the internal dielectric coating: $d / a_{1}=10^{-3}$, which for $a_{1}=2 \mathrm{~mm}$ means

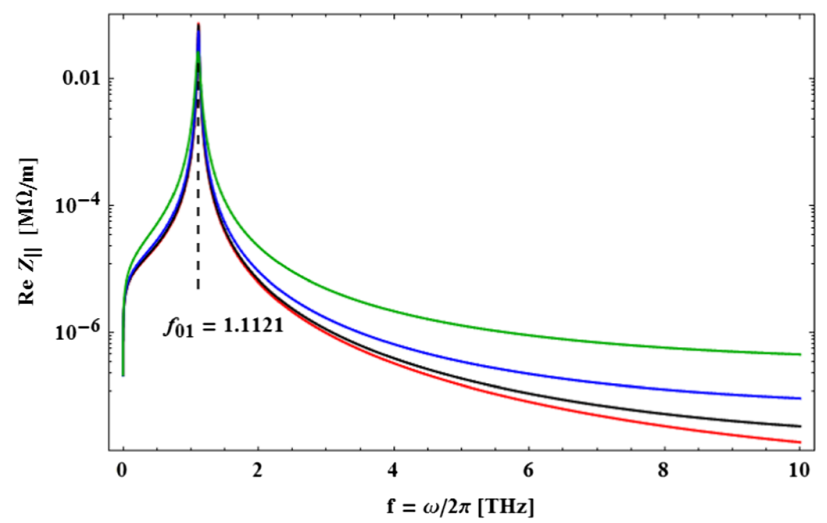

$d=2 \mu \mathrm{m}$. The real part of the relative dielectric constant is still equal to $\varepsilon_{1}^{\prime}=10$, and the outer wall of the waveguide is copper.

In this case, only a single resonant frequency is found (Fig. 10), corresponding to the fundamental $\mathrm{TM}_{01}$ mode. Moreover, the resonant frequency decreases with increasing losses in the dielectric (Fig. 10, right). The phase velocity of this mode at the resonance frequency coincides with the velocity of the particle (Fig. 11). As a result, the wakefield turns out to be fully monochromatic, as depicted in Fig. 12.

A smaller dielectric thickness also reduces the total loss of the lossy dielectric. Figure 12 illustrates the wakefield amplitude for a thin dielectric layer with low dielectric losses: In these cases $\left(\varepsilon_{1}^{\prime \prime}=0,0.1,0.5\right)$, the decrease in the amplitude of the wake function is mainly due to the finite conductivity of the outer wall; however, high-loss dielectrics $\left(\varepsilon_{1}^{\prime \prime}=3\right)$ can also provide a damping contribution.

As follows from a comparison of Figs. 2 and 10, a smaller dielectric thickness of the inner coating permits higher resonant frequencies. In particular, with $d=200 \mu \mathrm{m}$ the

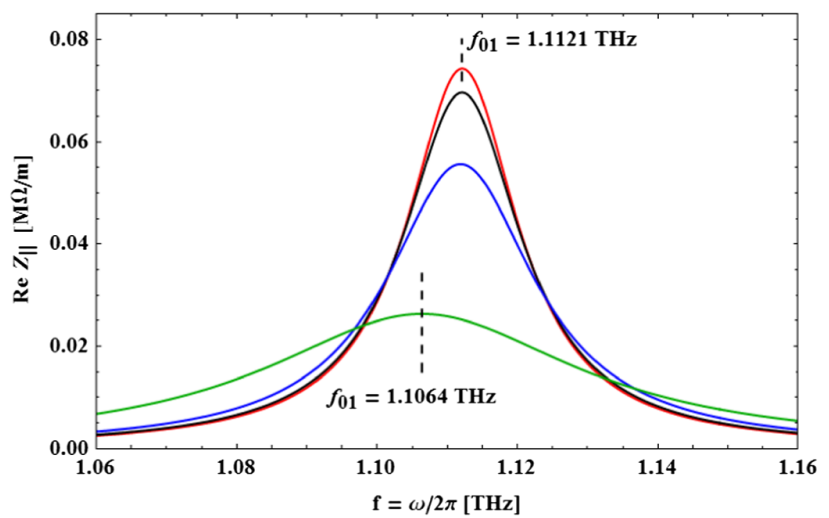

FIG. 10. The longitudinal monopole impedance of a two-layer metal-dielectric structure for different values of $\varepsilon_{1}^{\prime \prime}: \varepsilon_{1}^{\prime \prime}=0$ (red line), 0.1 (black line), 0.5 (blue line), and 3 (green line). Left: Wide terahertz range of impedance distribution. Right: The resonance neighborhood. 

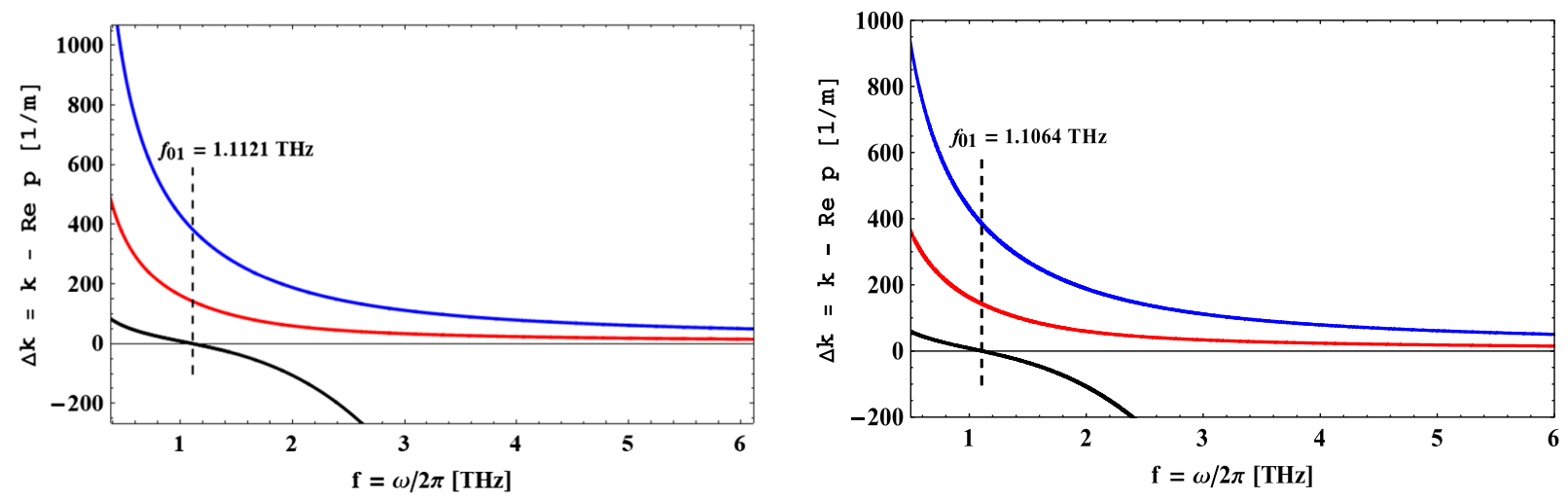

FIG. 11. Dispersion curves for the $\mathrm{TM}_{01}$ (black line), $\mathrm{TM}_{02}$ (red line), and $\mathrm{TM}_{03}$ (blue line) modes: $\varepsilon_{1}^{\prime \prime}=0$ (left) and $\varepsilon_{1}^{\prime \prime}=3$ (right). Synchronous frequencies correspond to $\Delta k=0$, which is reached only for the $\mathrm{TM}_{01}$ mode.

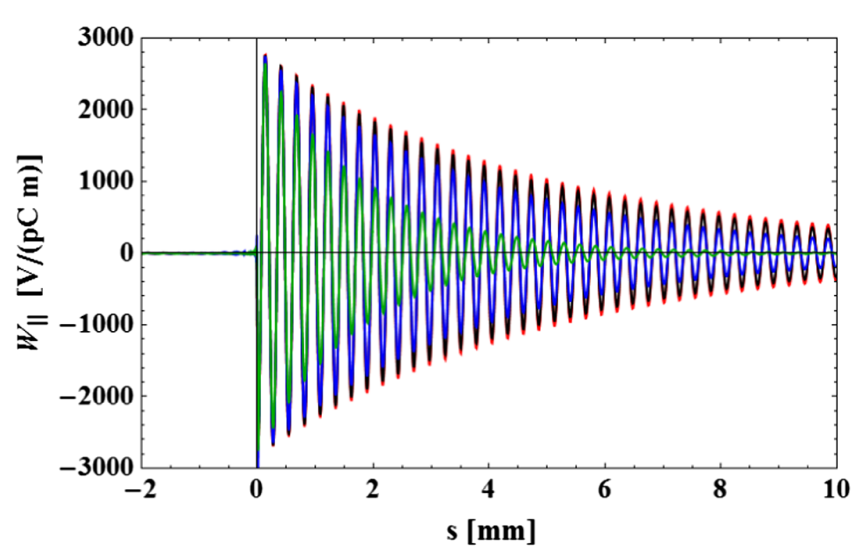

FIG. 12. Wake functions $\left(\varepsilon_{1}^{\prime}=10\right)$ for the cases $\varepsilon_{1}^{\prime \prime}=0$ (red line), 0.1 (black line), 0.5 (blue line), and 3 (green line); $a=2 \mathrm{~mm}$ and $d=2 \mu \mathrm{m}$.

main resonant frequency is in the subterahertz region (Fig. 2), while with $d=2 \mu \mathrm{m}$ it reaches to terahertz frequencies (Fig. 10).

For a thin lossless inner dielectric layer $\left(\varepsilon_{1}^{\prime \prime}=0\right)$ with an ideally conducting outer wall, the fundamental resonant frequency is determined by the expression [4,5]

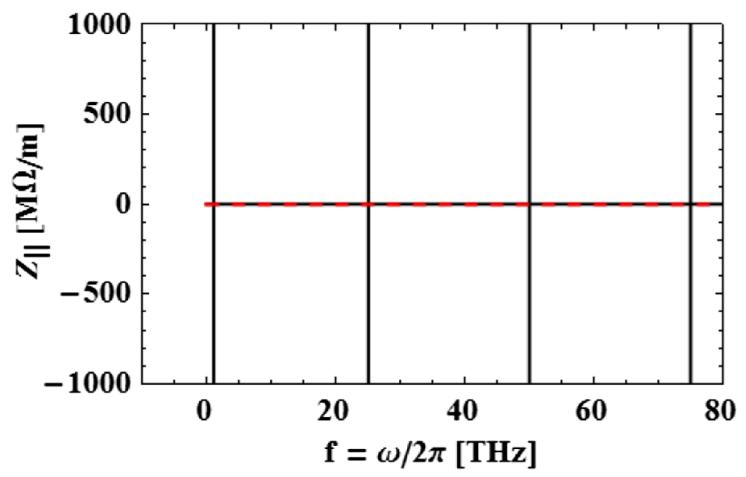

$$
f_{\text {res }}=\frac{c}{2 \pi} \sqrt{\frac{\varepsilon_{1}^{\prime}}{\varepsilon_{1}^{\prime}-1} \frac{2}{a_{1} d}} .
$$

The resonant frequency calculated using this formula for the selected parameters gives $f_{\text {res }}=1.1254 \mathrm{THz}$, whereas the same frequency calculated for the copper wall using the exact formulas gives a slightly lower value of $f_{\text {res }}=1.1121 \mathrm{THz}$ (see Fig. 10). The difference $\Delta f_{\text {res }}=13.2 \mathrm{GHz}$ is due to the finite conductivity of the outer copper wall. However, the structure with finite conductivity of the outer layer and thin internal dielectric layer has only the single synchronous $\mathrm{TM}_{01}$ mode (Fig. 13, right), while the same structure with the perfectly conducting outer wall has an infinite number of synchronous modes, as demonstrated in Fig. 13, left.

Note that the impedance of the perfectly conducting waveguide with the lossless dielectric internal layer has only an imaginary component and even with a sufficiently thin internal dielectric layer contains an infinite number of diverging resonant peaks (Fig. 13, left). It is noteworthy that the presence of even a small imaginary component in the dielectric constant of the layer substantially deforms the shape of the impedance curve: The impedance peaks

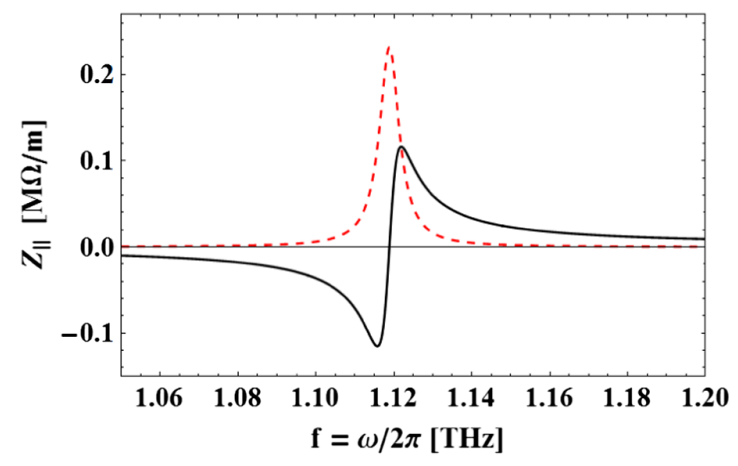

FIG. 13. The real (red, dashed line) and imaginary (black, solid line) components of the longitudinal impedance of the lossless thin dielectric loaded waveguide with a perfectly conducting wall (left) and with finite conducting (copper) walls (right); $a_{1}=2 \mathrm{~mm}$, $d=2 \mu \mathrm{m}$, and $\varepsilon_{1}^{\prime}=10$. 


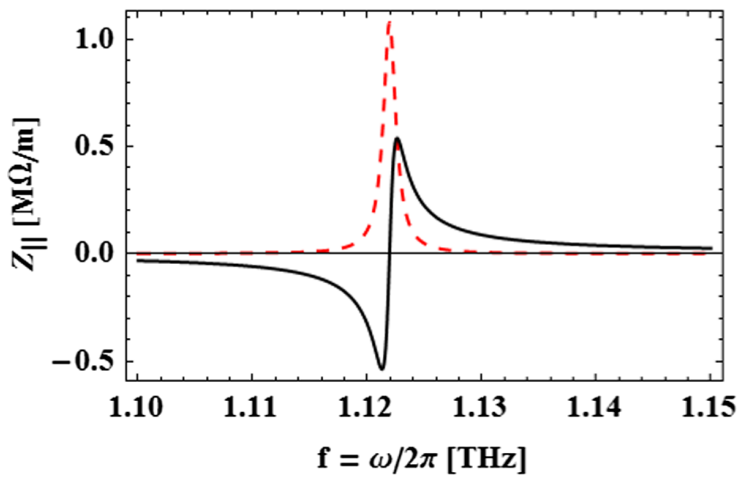

FIG. 14. Real (red, dashed line) and imaginary (black, solid line) components of the longitudinal impedance of a perfectly conducting waveguide with a thin dielectric layer: $a_{1}=2 \mathrm{~mm}$, $d=2 \mu \mathrm{m}, \varepsilon_{1}^{\prime}=10$; and $\varepsilon_{1}^{\prime \prime}=0.1$.

become finite, and their amplitudes decrease with increasing frequency. With a sufficiently small thickness of the inner layer, the impedance becomes single resonant with proportional real and imaginary parts (Fig. 14). The corresponding wake function is shown in Fig. 15.

The wake function is quasiperiodic, which indicates the monochromaticity of the wave emitted by the particle. Because of energy dissipation in the dielectric, the amplitude of the oscillations decreases slightly with increasing distance from the particle.

It can be shown [38] that, for the metal-dielectric structure with a thin dielectric layer and high conductive outer wall $\left(d / a_{1} \ll 1\right.$ and $\left.\sigma_{2} \gg 1\right)$, the amplitude of the real component of the impedance (1) takes the maximum value for the case

$$
d / a_{1}=3 /\left\{2\left(\varepsilon_{1}^{\prime 2}-2 \varepsilon_{1}^{\prime}+\varepsilon_{1}^{\prime \prime 2}\right)\right\} .
$$

For example, for $\varepsilon_{1}^{\prime \prime} \rightarrow 0$ and $d / a_{1}=0.1$ condition (18) corresponds to a dielectric constant of $\varepsilon_{1}^{\prime} \approx 5$ and for $d / a_{1}=10^{-3}$ to a dielectric constant of $\varepsilon_{1}^{\prime} \approx 40$.

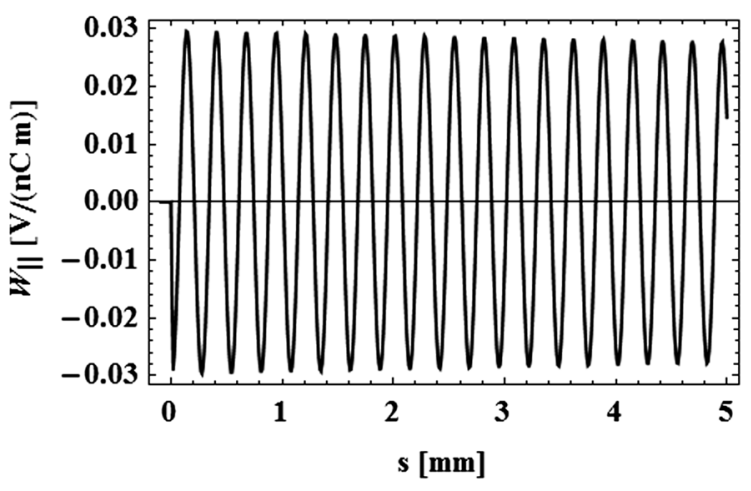

FIG. 15. Longitudinal monopole wake function of a perfectly conducting waveguide with a thin inner dielectric layer; $a_{1}=2 \mathrm{~mm}, d=2 \mu \mathrm{m}, \varepsilon_{1}^{\prime}=10$; and $\varepsilon_{1}^{\prime \prime}=0.1$.

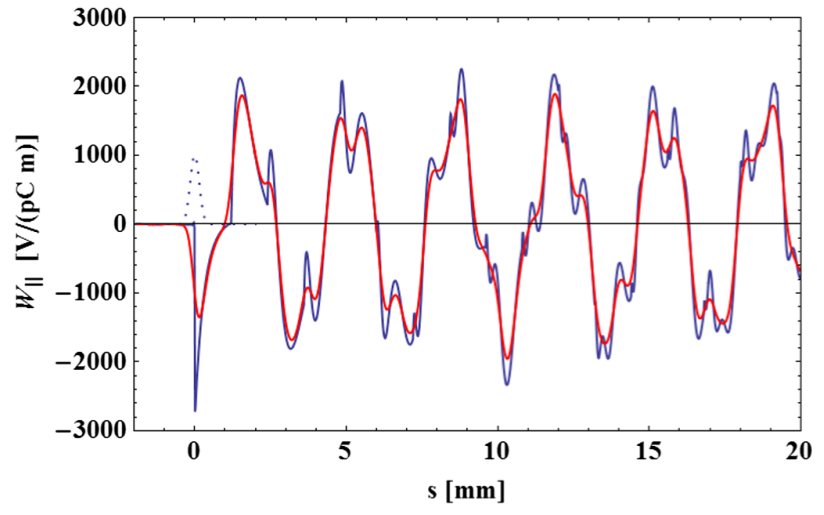

FIG. 16. Comparison of wake functions of a Gaussian bunch $(\sigma=0.5 \mathrm{ps}$, red line) and a point particle $(\sigma=0$, blue line); $\varepsilon_{1}^{\prime \prime}=0, a=2 \mathrm{~mm}$, and $d=200 \mu \mathrm{m}$. The dotted line indicates the bunch shape.

\section{THE GAUSSIAN BUNCH CASE}

In conclusion, we consider the transformation of the wake function in the transition from a point particle to a Gaussian bunch of charged particles. A long bunch, of course, suppresses higher harmonics (the high-frequency part of the impedance distribution). However, this is not always the case, as illustrated by the example shown in Fig. 16. Here, the wake function of a Gaussian bunch with an rms length of $0.5 \mathrm{ps}$ is superimposed on the wake function of a single particle in a waveguide with an internal coating of $200 \mu \mathrm{m}$ thickness (Fig. 5, blue).

On the above example, the presence of a 0.5 ps length bunch only partially suppresses the contributions from higher modes, which in practice leads to a slight smoothing of separate bursts of the point particle wake function, but does not completely eliminate the effect of the higher resonant frequencies. Thus, in a waveguide with a thick $(200 \mu \mathrm{m})$ dielectric coating, the presence of a sufficiently long beam $(0.5 \mathrm{ps})$ also does not lead to a wake function monochromatization. This serves as an additional argument for the use of a thin coating, of the order of a few microns, where the initial wake function of a point particle is already monochromatic and, therefore, the structure is single mode.

\section{CONCLUSION}

This work is devoted to the investigation of the resonance properties of a two-layer metal-dielectric cylindrical waveguide. In contrast to the generally accepted methodology, where the outer layer is a perfectly conducting metal and the inner dielectric layer has no dissipation, here the finite conductivity of the metal and losses in the dielectric layer are taken into account. Based on the results of the simultaneous analysis of the dispersion relations of azimuthally symmetric TM modes, the longitudinal monopole impedances, and wake potentials, new regularities for the formation of the radiation field of point charges in 
waveguides are established. In the case of a thin inner dielectric layer, updated mechanisms and new opportunities for establishing a single-mode regime with a slowly propagating fundamental $\mathrm{TM}_{01}$ mode are determined.

[1] S. Frankel, $\mathrm{TM}_{01}$ mode in cylindrical waveguide with two dielectrics, J. Appl. Phys. 18, 650 (1947).

[2] G. C. Bruck and E. R. Wicher, Slow transverse magnetic modes in cylindrical waveguides, J. Appl. Phys. 18, 766 (1947).

[3] R. B. R. Shersby-Harvie, A proposed new form of dielectric-loaded wave-guide for linear electron accelerators, Nature (London) 162, 890 (1948).

[4] A. V. Burov and A. V. Novokhatski, Wake potential of dielectric canal, in Preprint 92-17, Budker Institute of Nuclear Physics (Institute of Nuclear Physics, Novosibirsk, Russia, 1992), p. 18, http://wwwnew.inp.nsk.su/images/ preprint/1992_017.pdf.

[5] A. Novokhatski and A. Mosnier, Wakefields of short bunches in the canal covered with thin dielectric layer, in Proceedings of the 1997 Particle Accelerator Conference, Vancouver, BC, Canada, 1997 (IEEE, Piscataway, NJ, 2002), (Cat. No. 97CH36167), pp. 1661-1663, https://ieeexplore.ieee .org/document/750793?section=abstract.

[6] E. A. Nanni, W. R. Huang, K.-H. Hong, K. Ravi, A. Fallahi, G. Moriena, R. J. D. Miller, and F. X. Kärtner, Terahertz-driven linear electron acceleration, Nat. Commun. 6, 8486 (2015).

[7] F. Lemery, K. Floettmann, P. Piot, F. G. Kartner, and R. Aßmann, Synchronous acceleration with tapered dielectriclined waveguides, Phys. Rev. Accel. Beams 21, 051302 (2018).

[8] G. Burt, R. Letizia, C. Paoloni, S. P. Jamison, Y. Saveliev, R. B. Appleby, D. M. Graham, T. H. Pacey, H. Owen, G. Xia, A. W. Cross, A. Phelps, and K. Ronald, Dielectric and thz acceleration (data) programme at the Cockcroft institute, in Proceedings of 28th Linear Accelerator Conference (LINAC2016), East Lansing, MI, USA, 2016 (JACoW, Geneva, Switzerland, 2016), pp. 62-64, http:// epaper.kek.jp/linac2016/papers/proceed.pdf.

[9] A. L. Healy, G. Burt, S. P. Jamison, R. Valizadeh, M. J. Cliffe, and D. M. Graham, Design of a dielectric-lined waveguide for terahertz-driven linear electron acceleration, in Proceedings of 28th Linear Accelerator Conference (LINAC2016), East Lansing, MI, 2016 (JACoW, Geneva, Switzerland, 2016), pp. 158-160, http://epaper.kek.jp/linac 2016/papers/proceed.pdf.

[10] S. Antipov, C. Jing, M. Fedurin, W. Gai, A. Kanareykin, K. Kusche, P. Schoessow, V. Yakimenko, and A. Zholents, Experimental Observation of Energy Modulation in Electron Beams Passing through Terahertz Dielectric Wakefield Structures, Phys. Rev. Lett. 108, 144801 (2012).

[11] S. Antipov, S. V. Baryshev, R. Kostin, S. Baturin, J. Qiu, C. Jing, C. Swinson, M. Fedurin, and D. Wang, Efficient extraction of high power $\mathrm{THz}$ radiation generated by an ultra-relativistic electron beam in a dielectric loaded waveguide, Appl. Phys. Lett. 109, 142901 (2016).
[12] B. D. O'Shea, G. Andonian, S. K. Barber, C. I. Clarke, P. D. Hoang, M. J. Hogan, B. Naranjo, O. B. Williams, V. Yakimenko, and J. B. Rosenzweig, Conductivity Induced by High-Field Terahertz Waves in Dielectric Material, Phys. Rev. Lett. 123, 134801 (2019).

[13] Wei Gai, M. E. Conde, R. Konecny, and J. G. Power, Experimental demonstration of two beam acceleration using dielectric step-up transformer, in Proceedings of the 19th Particle Accelerator Conference, Chicago, IL, 2001 (IEEE, Piscataway, NJ, 2001) (Cat. No. 01CH37268), Vol. 3, p. 1880-1882.

[14] J. Shao, C. Jing, E. Wisniewski, J. Power, M. Conde, W. Liu, L. Zheng, N. Neveu, D. Doran, C. Whiteford, and W. Gai, Recent progress of short pulse dielectric two-beam acceleration, in Proceedings of the 9th Particle Accelerator Conference (IPAC2018), Vancouver, BC, Canada, 2018 (JACoW, Geneva, Switzerland, 2018), pp. 640-643, https://accelconf.web.cern.ch/ipac2018/.

[15] W. Gai, M. Conde, J. G. Power, and C. Jing, Considerations for a dielectric-based two-beam accelerator linear collider, in Proceedings of the International Particle Accelerator Conference, Kyoto, Japan (ICR, Kyoto, 2010), pp. 3428-3430.

[16] K.-Y. Ng, Wake fields in a dielectric-lined waveguide, Phys. Rev. D 42, 1819 (1990).

[17] S. Bettoni, P. Craievich, A. A. Lutman, and M. Pedrozzi, Temporal profile measurements of relativistic electron bunch based on wakefield generation, Phys. Rev. Accel. Beams 19, 021304 (2016).

[18] F. Lemery, K. Floettmann, T. Vinatier, and R. W. Assmann, A transverse deflecting structure with dielectric-lined waveguides in the sub-thz regime, in Proceedings of the 8th Particle Accelerator Conference (IPAC2017), Copenhagen, Denmark 2017 (JACoW, Geneva, Switzerland, 2017), pp. 215-218, https://accelconf.web.cern.ch/ ipac2017/papers/mopab052.pdf.

[19] V. V. Paramonov and K. Floettmann, Fundamental characteristics of transverse deflecting fields, Report No. 18-103, DESY, Hamburg, 2018.

[20] K. Floettmann and V. Paramonov, Beam dynamics in transverse deflecting rf structures, Phys. Rev. Accel. Beams 17, 024001 (2014).

[21] A. L. Healy, G. Burt, and S. P. Jamison, Electron-terahertz interaction in dielectric-lined waveguide structures for electron manipulation, arXiv:1802.00632.

[22] F. Lemery, P. Piot, G. Amatuni, P. Boonpornprasert, Y. Chen, J. Good, B. Grigoryan, M. Gross, M. Krasilinikov, O. Lishilin, G. Loisch, A. Oppelt, S. Philipp, H. Qian, Y. Renier, F. Stephan, and I. Zagorodnov, Passive Ballistic Microbunching of Nonultrarelativistic Electron Bunches Using Electromagnetic Wakefields in DielectricLined Waveguides, Phys. Rev. Lett. 122, 044801 (2019).

[23] A. Tsakanian, J. Rossbach, and M. Ivanyan, Longitudinal and transverse impedances of XFEL kicker vacuum chamber, in Proceedings of the 11th European Particle Accelerator Conference, Genoa, 2008 (EPS-AG, Genoa, Italy, 2008), pp. 700-702.

[24] M. Ivanyan, A. Grigoryan, A. Tsakanian, and V. Tsakanov, Narrow-band impedance of a round metallic pipe with a 
low conductive thin layer, Phys. Rev. Accel. Beams 17, 021302 (2014).

[25] A. Novokhatski, The effect of an oxide layer on resistivewall wake fields, in Proceedings of the 23rd Particle Accelerator Conference, Vancouver, Canada, 2009 (IEEE, Piscataway, NJ, 2009), pp. 4743-4745.

[26] M. I. Ivanyan, V. A. Danielyan, B. A. Grigoryan, A. H. Grigoryan, A. V. Tsakanian, V. M. Tsakanov, A. S. Vardanyan, and S. V. Zakaryan, High frequency single mode traveling wave structure for particle acceleration, Nucl. Instrum. Methods Phys. Res., Sect. A 829, 187 (2016).

[27] M. I. Ivanyan, V.S. Avagyan, V. A. Danielyan, A. V. Tsakanian, A. S. Vardanyan, and V. S. Zakaryan, On the resonant behavior of laminated accelerating structures, J. Instrum. 12, P03019 (2017).

[28] M. I. Ivanyan, A. Grigoryan, S. Zakaryan, and A. V. Tsakanian, Impedance and wake of two-layer metallic flat structure, Arm. J. Phys. 10, 36 (2017).

[29] K. L. F. Bane and G. Stupakov, Corrugated pipe as a beam dechirper, Nucl. Instrum. Methods Phys. Res., Sect. A 690, 106 (2012).

[30] G. V. Stupakov, Surface roughness impedance, SLAC Report No. SLAC-PUB-8743, 2000, https://www.slac .stanford.edu/cgi-bin/getdoc/slac-pub-8743b.pdf.
[31] G. Stupakov and K. L. F. Bane, Surface impedance formalism for a metallic beam pipe with small corrugations, Phys. Rev. Accel. Beams 15, 124401 (2012).

[32] M. Ivanyan and V. Tsakanov, Coupling impedance of rough resistive pipe, in Proceedings of the 2nd International Particle Accelerator Conference, San Sebastián, Spain (EPS-AG, Spain, 2011), pp. 1712-1714.

[33] TehTab.ru (in Russian), https://tehtab.ru/Guide/Guide Physics/ElectricityAndMagnethism/DEPLiquids/Dielectric ConstanVarious/.

[34] http://www.ydic.co.jp/english/technology/table_E.html\# Anchor_01.

[35] M. Ivanyan, E. Laziev, A. Tsakanian, V. Tsakanov, A. Vardanyan, and S. Heifets, Multi-layer tube impedance and external radiation, Phys. Rev. Accel. Beams 11, 084001 (2008).

[36] A. W. Chao, Physics of Collective Beam Instabilities in High Energy Accelerators (Wiley, New York, 1993).

[37] M. Ivanyan, A. Grigoryan, A. Tsakanian, and V. Tsakanov, Narrow-band impedance of a round metallic pipe with a low conductive thin layer, Phys. Rev. Accel. Beams 17, 021302 (2014).

[38] M. Ivanyan et al., On the resonant properties of THz metaldielectric waveguide impedance, in Proceedings of the 4th EAAC workshop, Italy, 2019, https://agenda.infn.it/event/ $17304 /$. 